\title{
Periprostatic fat is a risk factor for prostate cancer detection
}

Novel research from the University of Toronto reveals the potential utility of measuring periprostatic fat (PPF) for prostate cancer diagnosis. "Our group is the first to look at periprostatic fat thickness in a population of patients not yet diagnosed with prostate cancer," says Bimal Bhindi, lead author of the study. "This allowed us to identify PPF quantity as a risk factor for both the diagnosis of prostate cancer and for detection of high-grade disease on prostate biopsy."

A link between obesity and prostate cancer has been proposed previously, but the data are conflicting. Emerging evidence suggests that adipose tissue is an active endocrine organ, which prompted researchers to examine the local interactions between PPF and the prostate.

Investigators identified 931 men who underwent transrectal ultrasonography (TRUS)-guided biopsy for suspected prostate cancer from their institution's database. PPF thickness was measured on TRUS images as the shortest perpendicular distance between the prostate and pubic bone.

Multivariable analysis revealed that, after adjusting for other factors, the probability of detecting prostate cancer on biopsy increased with PPF thickness-by $12 \%$ per millimeter (OR 1.12, 95\% CI $1.02-1.23)$. Furthermore, they found that the odds increased by $20 \%$ per millimeter for high-grade (Gleason pattern $>3$ )

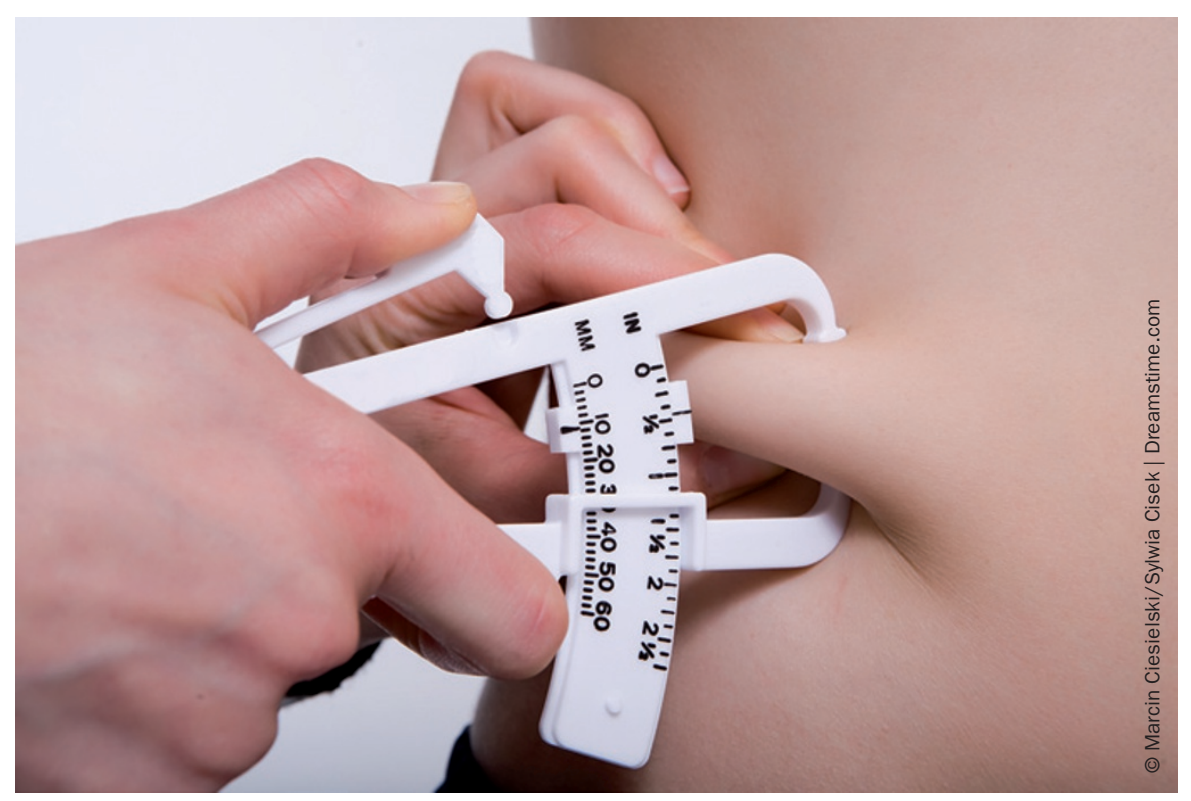

prostate cancer (OR 1.20, 95\% CI 1.071.34). Researchers also found that the area under the curve for PPF thickness was comparable to other clinical predictors of prostate cancer, including age, digital rectal examination and PSA level.

As Bhindi points out, the findings of this study highlight two key issues. "The first is whether PPF measurement might have a clinical role in prostate cancer risk assessment in the future. Further prospective studies are needed on this topic," says Bhindi. "Second, what are the potential cellular and molecular mechanisms underlying the association described in our study? Although still preliminary, we have begun to study the relationship between PPF thickness and adipokines, which may allow us to infer which signaling molecules have an important role."

Sarah Payton

Original article Bhindi, B. et al. Measurement of periprostatic fat thickness using transrectal ultrasonography (TRUS): a new risk factor for prostate cancer. BJU Int. doi:10.1111/j.1464-410X.2012.10957.x 